\title{
Efficient CTL productivity of modified fusion cells by increase of heat shock protein 70
}

\author{
TAIHEI KOIDE, HISAE IINUMA and RYOJI FUKUSHIMA \\ Department of Surgery, Teikyo University School of Medicine, Tokyo 173-0003, Japan
}

Received October 1, 2008; Accepted October 24, 2008

DOI: 10.3892/or_00000279

\begin{abstract}
In various loading methods of whole tumor cells or their derivatives, cells fusion of dendritic cells (DCs) and tumor cells have some advantages for antigen presentation, and could up-regulate cytotoxic T cells (CTL) for multiple tumor antigens, including unknown antigens. However, the mechanisms of strong CTL productivity of fusion cells (FCs) are still unknown. Heat shock proteins (HSPs) are molecular chaperones that cross-present chaperoned antigenic peptides with MHC class I molecules. Herein, we focused on clarifying the potential of FCs for CTL production from the comparison of DCs pulsed with two kinds of tumor cell lysates, such as soluble tumor cell lysates or freeze-thawed tumor cell lysates. DCs, CD8 ${ }^{+} \mathrm{T}$ cells and tumor cells were prepared from ten patients with gastric cancer, and paired autologous tumor cells were used in all experiments. FCs of OK432-treated DCs and heat-stressed tumor cells (modified FCs) showed significant up-regulation of tumor-associated CEA and HER-2 antigen, and DC-related HLA-DR and co-stimulatory molecules (CD83 and CD86). FCs showed significantly higher IFN- $\gamma$ and CTL productivity of $\mathrm{CD}^{+} \mathrm{T}$ cells than DCs pulsed with soluble or freeze-thawed tumor cell lysates. IFN- $\gamma$ and CTL productivity of FCs was significantly increased by the heat stress of tumor cells. HSP70 mRNA levels and production of HSP70 protein of modified FCs increased significantly as compared with those of DCs pulsed with soluble or freeze-thawed tumor cell lysates. DCs pulsed with HSP70.PC extracted from modified FCs could enhance CTL productivity significantly more than that of DCs pulsed with HSP70.PC from soluble or freezethawed tumor cell lysate pulsed-DCs. The significant upregulation of HSP70 mRNA and protein levels of modified FCs was related to the potential of CTL productivity. These
\end{abstract}

Correspondence to: Dr Hisae Iinuma, Department of Surgery, Teikyo University School of Medicine, 2-1-1, Kaga, Itabashi-ku, Tokyo 173-0003, Japan

E-mail: iinuma@med.teikyo-u.ac.jp

Abbreviations: HSP, heat shock protein; DC, dendritic cell; FC, fusion cell; TLR, toll-like receptor; PC, peptide complex

Key words: fusion cell, cytotoxic T lymphocyte, heat shock protein 70 , gastric cancer results suggest that modified FCs possess stronger ability for MHC-restricted CTL production than DCs loaded with soluble or freeze-thawed tumor cell lysates.

\section{Introduction}

Dendritic cells (DCs) are specialized antigen-presenting cells with the ability to initiate a strong primary immune response (1). DCs loaded with tumor-associated antigen can effectively stimulate host immune responses to syngeneic tumor cells. Although many methods for the preparation of tumorassociated antigen have been examined, there are two general approaches for loading tumor-associated antigens: the use of a defined single tumor-associated antigen, such as a peptide, and the use of whole tumor cells or their derivatives for the induction of polyclonal CTLs to multiple antigens, including the unknown antigens. The loading of DCs with defined peptides seems to be a logical approach in the design of specific tumor vaccines; however, because of its dependence on the efficacy of antigen loading and availability of chemically defined antigens, peptides have significant limitations, such as the human leukocyte antigen (HLA) restriction as a source of tumor antigens. In contrast, whole tumor cells or their derivatives are interesting because of no restriction by HLA typing to increase patients receiving a DCbased tumor vaccine. Various methods of antigen preparation using whole tumor cells have been attempted, such as fusion cells (FCs) of DCs and tumor cells, irradiated tumor cells, tumor lysates, RNA extracted from tumor cells $(2,3)$. Using the mouse model, a few studies reported the comparison of the antigen loading method $(4,5)$. However, there is still considerable controversy as to which antigen-loading methods represent the most immunogenic forms of vaccines that are capable of eliciting clinical relevant therapeutic immunity.

FCs made from DCs and tumor cells possess both cell components of tumor antigens and accessory molecules necessary for antigen processing and presentation. The protective and therapeutic effects of an FC vaccine have already been reported in animal models (6-9). In clinical trials, partial therapeutic effects have been demonstrated in patients with melanoma, renal carcinoma, and gastrointestinal cancer; however, the mechanisms of strong antigen presentation and the induction of anti-tumor immunity of FCs have not been defined (10-17). In a mouse model, it has been reported that heat shock protein 70 (HSP70) with peptide complex (HSP70.PC) derived from FCs possess strong immuno- 
genicity as a tumor vaccine (18). HSPs are molecular chaperones and play a significant role in the immune response when released from cells in complex with chaperoned antigenic peptides (17-20). DCs have a take-up process, and present exogenous antigens such as HSPs in association with MHC class I molecules. This process is termed crosspresentation. It has been demonstrated that HSPs gain access to the MHC class I-processing pathway and initiate cytotoxic $\mathrm{T}$ lymphocyte (CTL) responses $(21,22)$.

In this study, we compared the CTL productivities of FCs and DCs pulsed with tumor lysates, and clarified the potential of modified FCs of OK-432-stimulated DCs and heat-stressed tumor cells. In addition, the mechanism of CTL productivity was investigated focusing on the expression of HSP70.

\section{Materials and methods}

Cell lines and autologous tumor cells. The human gastric cancer cell lines MKN28 (HLA-A*31012, HER-2+) and leukemia cell line K562 (NK sensitive) were obtained from the American Type Culture Collection (Manassas, VA, USA). Cells were cultured in RPMI-1640 medium supplemented with $10 \%$ fetal calf serum (FCS) and $1 \%$ penicillin and streptomycin (Invitrogen Corp., CA, USA) at $37^{\circ} \mathrm{C}$ in $5 \% \mathrm{CO}_{2}$ atmosphere. Surgical specimens of ten patients with gastric cancer were obtained with informed consent and the approval of our institutional review board. Cell suspension of gastric cancer tissues and normal gastric tissues were prepared by treatment of enzyme mixture of collagenase $(0.02 \%)$, DNase $(0.02 \%)$ and Pronase $(0.05 \%)$, and maintained in AIM-V medium (Invitrogen Corp., NY, USA) supplemented with $10 \%$ heat-inactivated autologous serum, $2 \mathrm{mmol} / \mathrm{l} \mathrm{L-glutamine}$, $100 \mathrm{U} / \mathrm{ml}$ penicillin and $0.1 \mathrm{mg} / \mathrm{ml}$ streptomycin. For heat shock, cancer cells were warmed to $40^{\circ} \mathrm{C}$ for $3 \mathrm{~h}$ and incubated for $24 \mathrm{~h}$ at $37^{\circ} \mathrm{C}$ in $5 \% \mathrm{CO}_{2}$ for recovery (23). Then live cells were collected and used for cell fusion.

Preparation of DCs. Blood samples were obtained with the informed consent and approval of our institutional review board. Immature DCs were generated from peripheral blood mononuclear cells of patients with gastric cancer as described previously (24). For maturation, immature DCs were stimulated with $0.1 \mathrm{KE} / \mathrm{ml}(0.01 \mathrm{mg}$ of dried streptococci) OK-432 (Chugai Pharmaceutical Co., Tokyo, Japan) for $24 \mathrm{~h}$ (OK-DC) (24).

DCs pulsed with tumor soluble lysate or freeze-thawed tumor cells. Two kinds of tumor lysate, soluble and freeze-thawed tumor cell lysates were prepared. Soluble autologous tumor lysate was prepared as described previously (4). Briefly, heated or unheated tumor cells were homogenized and the extracts were centrifuged at 50,000 x g for $30 \mathrm{~min}$. The supernatant was dialyzed in phosphate-buffered saline and prepared as soluble lysate. For preparation of freeze-thawed tumor cell lysate, autologous heated or unheated tumor cells were stressed in four cycles of freezing and thawing. Soluble (Sol-Lysate) or freeze-thawed tumor cell lysate (FT-Lysate) was cultured with immature $\mathrm{DCs}$ at $37^{\circ} \mathrm{C}$ for $18 \mathrm{~h}$ (tumor cell: $\mathrm{DC}=2: 1$ ); then, stimulated with $0.1 \mathrm{KE} / \mathrm{ml} \mathrm{OK}-432$ for $24 \mathrm{~h}$ for DC maturation.
Preparation of fusion cells of DCs and autologous tumor cells. OK-432 stimulated DCs were fused with heated or unheated irradiated (100 Gy) autologous tumor cells at a ratio of 2:1 by the two-step fusion method described previously (7). To determine fusion efficiency, DCs and tumor cells were prestained with the fluorescent dyes $\mathrm{DiO}$ and DiI (Invitrogen), respectively, and analyzed with FACSCalibur flow cytometer and Cell Quest software (BD Biosciences, CA, USA).

Phenotype analysis. Cells were stained with FITC-conjugated monoclonal antibodies (mAbs) against CEA (MBL, Gunma, Japan), HER-2 (BD PharMingen, CA, USA), HLA-ABC, HLA-DR, CD80, CD83 and CD86 (Beckman Coulter Inc., CA, USA). CEA mAb were used after the treatment of IntraPrep (Beckman Coulter Inc.) to increase the membrane permeability. The expression of surface molecules was quantified using FACSCalibur and Cell Quest software.

Enzyme-linked immunospot (ELISPOT) assay. For the study of IFN- $\gamma$ productivities of FCs and tumor lysate-pulsed DCs, eight experimental groups were examined as follows: 1) OK432-treated DCs and unheated autologous tumor cells (FC), 2) FCs of OK-432-treated DCs and heated autologous tumor cells (HS/FC), 3) DCs pulsed with freeze-thawed autologous tumor lysate (FT-Lysate), 4) DCs pulsed with heat-stressed freeze-thawed autologous tumor lysate (HS/FT-Lysate), 5) DCs pulsed with autologous soluble tumor lysate (SolLysate), 6) DCs pulsed with heat-stressed autologous soluble tumor lysate (HS/Sol-Lysate), 7) Mixture of OK-432 treated DCs and heat-stressed autologous tumor cells (Mix/HSTumor/DC) and 8) medium. CD8 T cells were stimulated every 5 days by autologous FC or DC pulsed with autologous tumor lysate in complete medium (RPMI-1640 medium supplemented with at $25 \mathrm{mM}$ HEPES, $10 \%$ heat-inactivated FCS, 2 mM l-glutamine, $0.1 \mathrm{mM}$ nonessential amino acids, $1 \mathrm{mM}$ sodium pyruvate, $100 \mathrm{U} / \mathrm{ml}$ penicillin, $100 \mu \mathrm{g} / \mathrm{ml}$ streptomycin, $5 \times 10^{-5}$ M $2 \mathrm{ME}$ ) with 10 units of IL-2. After once or twice stimulation, $\mathrm{CD} 8^{+} \mathrm{T}$ cells that secrete $\mathrm{IFN}-\gamma$ were detected by ELISPOT assay kits (BD Pharmingen), and viable spots were enumerated using the ELISPOT reader (Carl Zeiss, Berlin, Germany).

Real-time RT-PCR of HSP70 mRNA. Total RNA was isolated using RNeasy spin column kits (Qiagen, Valencia, CA, USA), and cDNA was synthesized by SuperScript II RNase H reverse transcriptase (Invitrogen Corp., CA, USA). Real-time quantitative RT-PCR of HSP70 mRNA and glyceraldehydes3-phosphate-dehydrogenase (GAPDH) mRNA was performed using a StepOne (ABI, CA, USA). Primers and probes of HSP70 (Hs00382884) and GAPDH (Hs02786624) were purchased from Applied Biosystems (ABI). For amplification, initial denaturation at $95^{\circ} \mathrm{C}$ for $10 \mathrm{~min}$ was followed by 40 cycles of $15 \mathrm{sec}$ at $95^{\circ} \mathrm{C}$ and $1 \mathrm{~min}$ at $60^{\circ} \mathrm{C}$. All samples were measured in triplicate. The level of HSP was normalized by GAPDH, and the ratio of HSP to GAPDH was calculated.

ELISA for HSP70 protein mesurements. FCs, tumor lysatepulsed DCs and mixture of DC and tumor cells were cultured for 3 days. HSP70 protein levels of supernatants of these 
Table I. Characteristics of gastric carcinoma and percentage of DC/tumor FCs.

\begin{tabular}{|c|c|c|c|c|c|c|c|c|}
\hline \multirow[b]{2}{*}{ Patient list } & \multirow[b]{2}{*}{ HLA-ABC } & \multirow[b]{2}{*}{ HLA-A $* 24$} & \multirow[b]{2}{*}{ HLA-A $* 2$} & \multirow[b]{2}{*}{ HLA-DR } & \multirow[b]{2}{*}{ CEA } & \multirow[b]{2}{*}{ HER-2 } & \multicolumn{2}{|c|}{ Fusion efficiency (\%) } \\
\hline & & & & & & & $\mathrm{FC}$ & $\mathrm{HS} / \mathrm{FC}$ \\
\hline 1 & + & $+(2402101)$ & - & - & + & - & 50.2 & 61.2 \\
\hline 2 & + & $+(2402101)$ & - & - & + & - & 47.3 & 53.1 \\
\hline 3 & + & $+(2402101)$ & - & - & + & - & 43.8 & 50.7 \\
\hline 4 & + & $+(2402101)$ & - & - & + & + & 57.4 & 51.4 \\
\hline 5 & + & $+(2402101)$ & - & - & + & - & 55.2 & 47.6 \\
\hline 6 & + & $+(2402101)$ & - & - & + & + & 42.1 & 57.7 \\
\hline 7 & + & $+(2402101)$ & - & - & + & - & 38.7 & 60.1 \\
\hline 8 & + & - & $+(0201)$ & - & + & + & 57.1 & 62.5 \\
\hline 9 & + & - & $+(0207)$ & - & + & + & 46.2 & 55.7 \\
\hline 10 & + & - & $+(0207)$ & - & + & + & 48.4 & 55.8 \\
\hline Mean \pm SD & & & & & & & $48.6 \pm 6.4$ & $55.6 \pm 4.9$ \\
\hline
\end{tabular}

cells were tested by ELISA assay (StressXpress, Stressgen Biosciences, MI, USA).

Preparation of HSP70 by immunoprecipitation. The cells were incubated in lysis buffer (PBS with $1 \%$ Triton X-100, $1 \mathrm{mg} / \mathrm{ml} \mathrm{BSA}, 0.2 \mathrm{U} / \mathrm{ml}$ aprotinin, $1 \mathrm{mM}$ PMSF) with $20 \mathrm{U} / \mathrm{ml}$ apyrase (Sigma-Aldrich Japan, Tokyo, Japan) for $1 \mathrm{~h}$ on ice. The lysates were clarified by centrifugation and aqueous phases were collected. Anti-HSP70 mAb was added and incubated overnight at $4^{\circ} \mathrm{C}$, and then protein $\mathrm{A} / \mathrm{G}$ agarose was added and incubated at $4^{\circ} \mathrm{C}$ for an additional $1 \mathrm{~h}$. After extensive washing with lysis buffer, peptide complex with HSP70 was extracted (HSP70.PC), and a portion of sample was analyzed by Western blotting described previously (7). Then peptide complex with HSP70 was purified by gel filtration chromatography (GE Healthcare UK Ltd., Buckinghamshire, UK) for CTL assay. A portion of sample was analyzed by 12.5\% SDS-PAGE and silver staining (EzStain Silver, ATTO Co., Tokyo, Japan) (23).

CTL assay. CTL assays were examined for two purposes of the experiment. The first purpose was examination of CTL productivity by stimulation with DC-based vaccines as follows: HS/FC, FC, Mix/HS-Tumor/DC and DCs pulsed with HS/FT-Lysate, FT-Lysate, HS/Sol-Lysate or Sol-Lysate. The second purpose was examination of CTL productivities by DCs pulsed with HSP70.PC purified from HS/FC, FC, HS/Tumor, Tumor, HS/FT-Lysate or HS/Sol-Lysate. In this study, immature DCs were pulsed with $1.25,2.5$ or $5 \mu \mathrm{g} / \mathrm{ml}$ of purified HSP70.PC for $24 \mathrm{~h}$, and then matured with 0.1 $\mathrm{KE} / \mathrm{ml} \mathrm{OK}-432$ for $24 \mathrm{~h}$. CD8 ${ }^{+} \mathrm{T}$ cells were stimulated every 7 days by autologous DCs pulsed with HSP70.PC. After two times of stimulation, $\mathrm{CD}^{+} \mathrm{T}$ cells were mixed with $\mathrm{Na}^{51} \mathrm{CrO}_{4}$-labeled target cells at various ratios in a 96-well microplate. As target cells, autologous gastric cancer cells, autologous gastric normal cells, allogeneic MKN28 cells and K562 cells were used. In the mAb-blocking assay, target cells were incubated with anti-MHC class I or class II mAb before the addition of effector cells. After incubation for $6 \mathrm{~h}$ at $37^{\circ} \mathrm{C}$ with $5 \% \mathrm{CO}_{2}$, radioactivity levels of supernatants were quantitated in a gamma-counter and specific cytotoxicity was calculated described previously (7). Each sample was performed in triplicate.

Statistical analysis. Multiple groups were evaluated by ANOVA and the post-hoc Scheffe multirange test. $\mathrm{p}<0.05$ was considered significant. Values are presented as the mean \pm standard deviation (SD).

\section{Results}

Comparison of phenotypes of tumor cells, DCs, lysate-pulsed $D C s$ and FCs. Tumor cells isolated from gastric cancer patients showed high expression levels of CEA, HER-2 and HLA-ABC molecules, but not HLA-DR, CD80, CD83 or CD86 molecules (Table I and Fig. 1A). Heat-stressed tumor cells (HS/Tumor) resulted in a significant up-regulation of CEA and HER-2 antigens. DCs stimulated with OK-432 expressed significantly higher levels of HLA-DR, CD83 and CD86 molecules as compared with those of immature DCs (Imm-DC) (Fig. 1B). Next, we examined the phenotypes of DCs pulsed with four types of cell lysate as follows: freezethawed tumor lysate (FT-Lysate), heat-stressed FT-Lysate (HS/FT-Lysate), soluble tumor lysate (Sol-Lysate) and heatstressed Sol-Lysate (HS/Sol-Lysate). DCs pulsed with four types of tumor lysates showed high levels of HLA-ABC, HLA-DR, and CD80, CD83, CD86 molecules and medium levels of CEA and HER-2 (Fig. 1C). There were no significant differences between DCs pulsed with Sol-Lysate and FTLysate. In contrast, FC of DC and tumor cells showed expressions of HLA-ABC, HLA-DR and co-stimulatory molecules from DCs, and CEA and HER-2 molecules from tumor cells (Fig. 1D). By OK432 stimulation of DC, expression levels of HLA-DR, CD83 and CD86 of FC increased significantly. Furthermore, FC of OK-treated DC and heat-stressed tumor cells (HS/FC) showed a significant up-regulation of CEA and HER-2 molecules as compared with those of nonheat-stressed FC. These findings suggest that the tumor antigen and co-stimulator molecules which are necessary for antigen presentation are expressed in FC, and FC modified by heat 

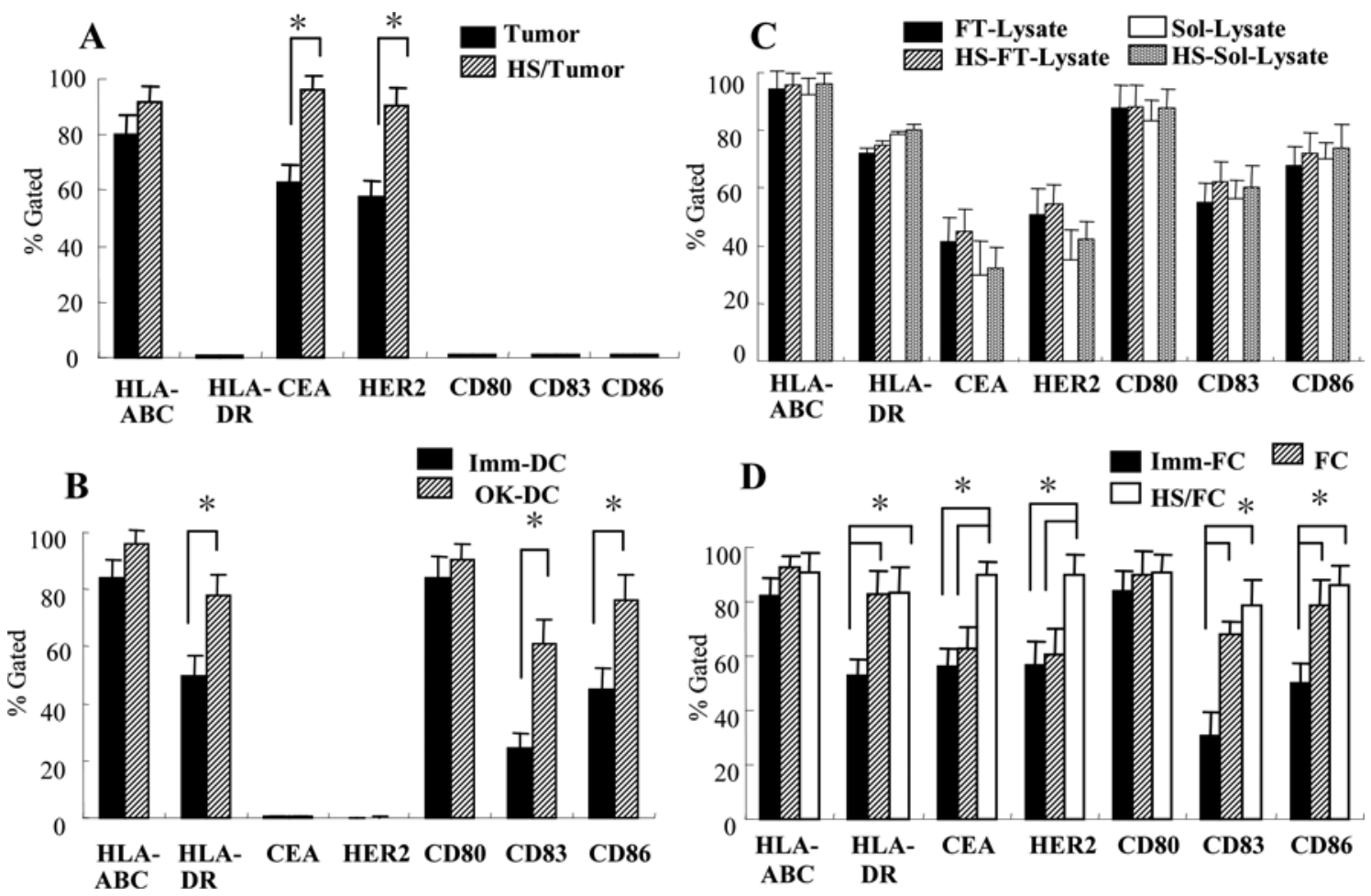

Figure 1. Characteristics of cell-surface phenotypes. (A) Cell-surface markers of unheated-autologous tumor cells (Tumor) and heated autologous tumor cells (HS/Tumor) were analyzed. (B) Cell-surface markers of immature DCs (Imm-DC) and OK432-treated DCs (OK-DC) were analyzed. (C) Cell-surface markers of DCs pulsed with freeze-thawed autologous tumor lysate (FT-Lysate), heat-stressed freeze-thawed autologous tumor lysate (HS/FT-Lysate), soluble autologous tumor lysate (Sol-Lystae) or heat-stressed soluble autologous tumor lysate (HS/Sol-Lysate), were analyzed. (D) Cell-surface markers of FC of immature DC and unheated autologous tumor cells (Imm-FC), FCs of OK432-treated DC and unheat-stressed autologous tumor cells (FC) and FC of OK432treated DC and heat-stressed autologous tumor cells (HS/FC). Cells were incubated with FITC-conjugated mAbs against HLA-ABC, HLA-DR, CD80, CD83, CD86, CEA or HER-2 and analyzed by flow cytometry. Mean \pm SD $(n=10),{ }^{*} \mathrm{p}<0.05$
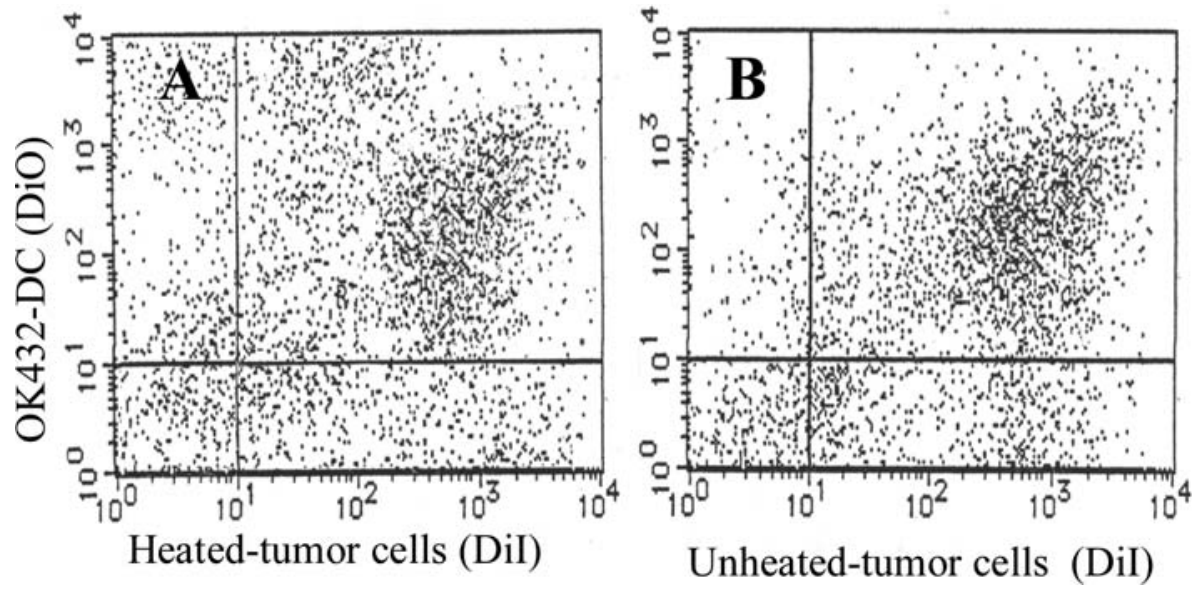

Figure 2. Fusion efficiency of DC and heated or unheated autologous tumor cells. Fusion efficiency of HS/FC (A) and FC (B) was examined by FACS. The presented FACS profile is representative of ten patients.

stress show increased expression levels of tumor antigens molecules.

Fusion efficiency. The average fusion efficiency of non-heatstressed autologous tumor cells and DC (FC) and heat-stressed autologous tumors and DCs (HS/FC) was $48.6 \pm 6.4 \%$ and $55.6 \pm 4.9 \%$, respectively with no significant differences between them (Table I). Fig. 2 shows the FACS profile of a representative patient.
IFN- $\gamma$ productivity of FC and tumor lysate-pulsed DC. IFN- $\gamma$ productivities of autologous $\mathrm{CD} 8^{+} \mathrm{T}$ cells by stimulation with FC (HS/FC and FC), mixture of autologous heat-stressed tumor cells and OK-stimulated DCs (Mix/HS-Tumor/DC), DC pulsed with two types of autologous tumor lysates, freezethawed tumor cells (HS/FT-Lysate, FT-Lysate) and soluble lysate (HS/Sol-Lysate, Sol-Lysate) (Fig. 3). On the first stimulation of DC loaded with tumor antigens, $\mathrm{CD}^{+} \mathrm{T}$ cells stimulated with $\mathrm{HS} / \mathrm{FC}$ or FC showed significantly high 

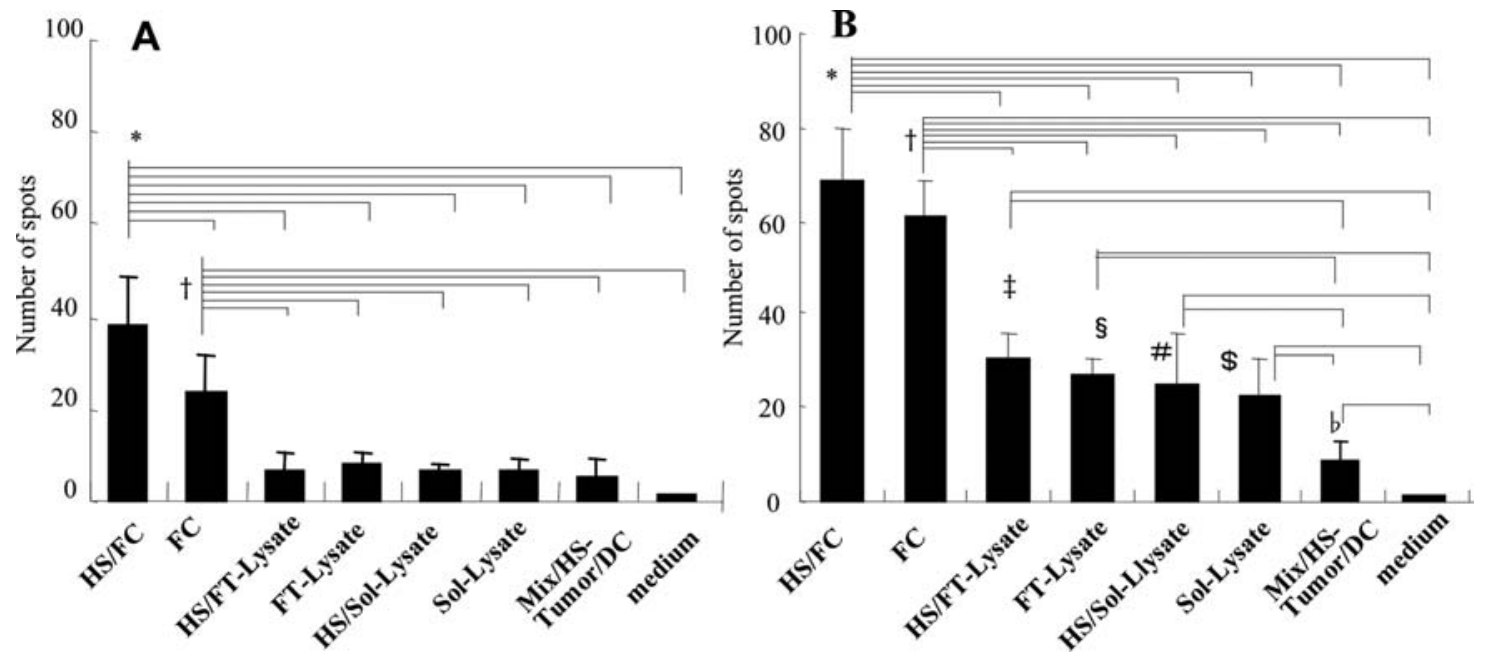

Figure 3. Comparison of IFN- $\gamma$ productivity by tumor antigen-loading methods. CD8 ${ }^{+} \mathrm{T}$ cells were stimulated once (A) or twice (B) with autologous HS/FC, FC, Mixture if HS-Tumor and OK-DC (Mix/HS-Tumor/DC) or autologous DC pulsed with HS/FT-Lysate, FT-Lysate, HS/Sol-Lysate or Sol-Lysate. The number of IFN- $\gamma$ production cells was examined by ELISpot assay. (A) ${ }^{*} \mathrm{p}<0.05$ (HS/FC vs. all other groups), ${ }^{\dagger} \mathrm{p}<0.05$ (FC vs. all other groups). (B) ${ }^{*} \mathrm{p}<0.05$ (HS/FC vs. all other groups), ${ }^{\dagger} \mathrm{p}<0.05$ (FC vs. all other groups), ${ }^{\star} \mathrm{p}<0.05$ (HS/FT-Lysate vs. Mix/HS-Tumor/DC and medium), ${ }^{8} \mathrm{p}<0.05$ (FT-Lysate vs. Mix/HSTumor/DC and medium), ${ }^{\#} \mathrm{p}<0.05$ (HS/Sol-Lysate vs. Mix/HS-Tumor/DC and medium), ${ }^{s} \mathrm{p}<0.05$ (Sol-lysate vs. Mix/HS-Tumor/DC and medium), ${ }^{b} \mathrm{p}<0.05$ (Mix/HS-Tumor/DC vs. medium). Mean $\pm \mathrm{SD}(\mathrm{n}=10)$.
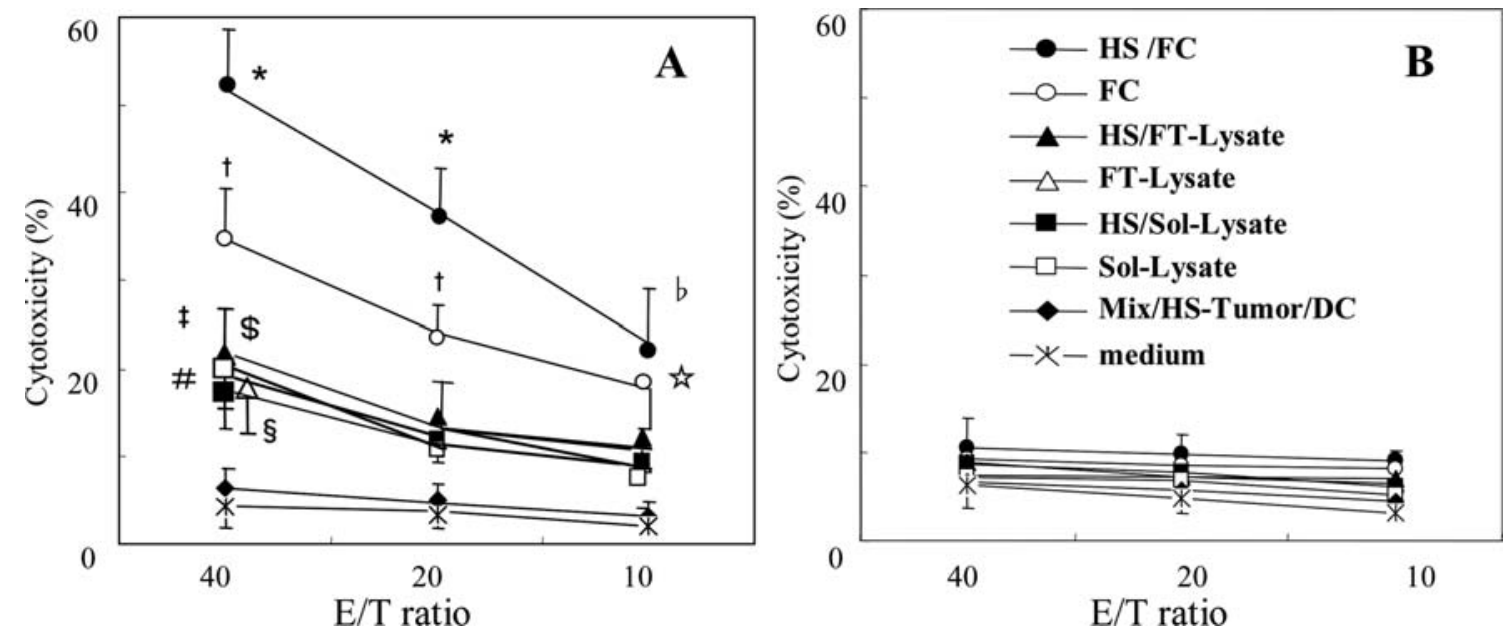

Figure 4. Comparison of CTL productivity by different tumor antigen-loading methods. CD8 ${ }^{+} \mathrm{T}$ cells were stimulated twice with autologous $\mathrm{HS} / \mathrm{FC}, \mathrm{FC}$ or autologous DCs pulsed with HS/FT-Lysate, FT-Lysate, HS/Sol-Lysate, Sol-Lysate, Mix/HS-Tumor/DC and medium. CTL activities against autologous tumor cells (A) or allogeneic MKN28 (B) were examined by ${ }^{51} \mathrm{Cr}$ release assay. ${ }^{*} \mathrm{p}<0.05$ (HS/FC vs. all other groups). ${ }^{\dagger} \mathrm{p}<0.05$ (FC vs. all other groups), ${ }^{*} \mathrm{p}<0.05$ (HS/FT-Lysate vs. Mix/HS-Tumor/DC and medium), ${ }^{8} \mathrm{p}<0.05$ (FT-Lysate vs. Mix/HS-Tumor/DC and medium), ${ }^{\#} \mathrm{p}<0.05$ (HS/Sol-Lysate vs. Mix/HSTumor/DC and medium), ${ }^{\$} \mathrm{p}<0.05$ (Sol-Lysate vs. Mix/HS-Tumor/DC and medium), ${ }^{\mathrm{b}}\left(\mathrm{HS} / \mathrm{FC}\right.$ vs. all groups except FC), ${ }^{\text {r }}$ (FC vs. Mix/HS-Tumor/DC and medium). Mean $\pm \operatorname{SD}(n=10)$.

levels of IFN- $\gamma$-positive spots as compared with those of other groups (Fig. 3A). In contrast, autologous $\mathrm{CD}^{+} \mathrm{T}$ cells stimulated with DC pulsed with freeze-thawed lysates or soluble lysates did not show significant levels of spots. By the second stimulation, spots of IFN- $\gamma$-positive $\mathrm{CD}^{+} \mathrm{T}$ cells stimulated with $\mathrm{HS} / \mathrm{FC}$ or FC were increased significantly more than those of HS/FT-Lysate, FT-Lysate, HS/SolLysate, Sol-Lysate, Mix/ HS-Tumor/DC and medium. CD8 ${ }^{+} \mathrm{T}$ cells stimulated with DC pulsed with freeze-thawed lysate or soluble lysate showed significantly higher levels of IFN- $\gamma$ positive spots than those of Mix/HS-Tumor/DC and medium. Heat-stressed tumor cells enhanced productivity of IFN- $\gamma$, and the HS/FC-stimulated group showed the highest number of spots in all groups. These results indicate that FC possess enhanced stimulatory ability of autologous $\mathrm{CD} 8^{+} \mathrm{T}$ cell IFN- $\gamma$ production as compared with tumor lysate-pulsed DC, and modifications of FC by heat stress could enhance this ability.

CTL productivity of FCs and tumor lysate-pulsed DCs. CD8 ${ }^{+}$ $\mathrm{T}$ cells from patients were stimulated twice with autologous DCs loaded with autologous tumor cells or tumor lysate, and their cytotoxicity against autologous tumor cells was examined (Fig. 4A). CTL activities induced by HS/FC were significantly higher than those of FC, HS/FT-Lysate, FTLysate, HS/Sol-Lysate, Sol-Lysate, Mix/HS-Tumor/DC and medium. The HS/FC group showed the highest cytotoxicity 


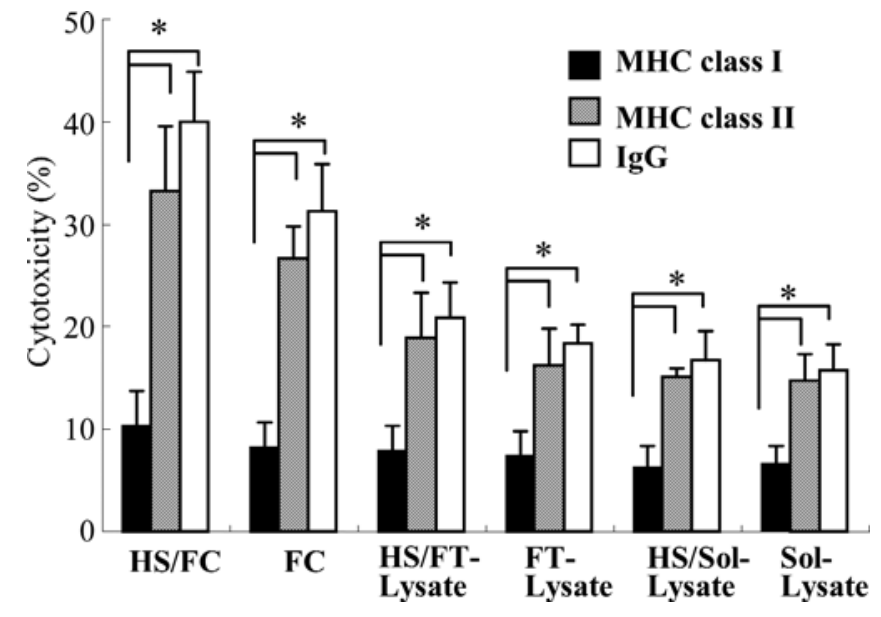

Figure 5. MHC restriction of CTL induced by FC or tumor lysate-pulsed DCs. CD8 ${ }^{+} \mathrm{T}$ cells from patients were co-cultured with autologous FC or tumor lysate-pulsed DCs (HS/FT-lysate, FT-lysate, HS/Sol-lysate, SolLysate) and cytotoxicity against autologous tumor cell was examined by ${ }^{51} \mathrm{Cr}$ release assay. Target cells were pretreated with anti-MHC class I mAb, anti-MHC class II mAb or IgG. The ratio of E:T cells were 50:1. ${ }^{*} \mathrm{p}<0.05$ (anti-MHC Class I vs. anti-MHC class II, IgG). Mean \pm SD $(n=10)$.
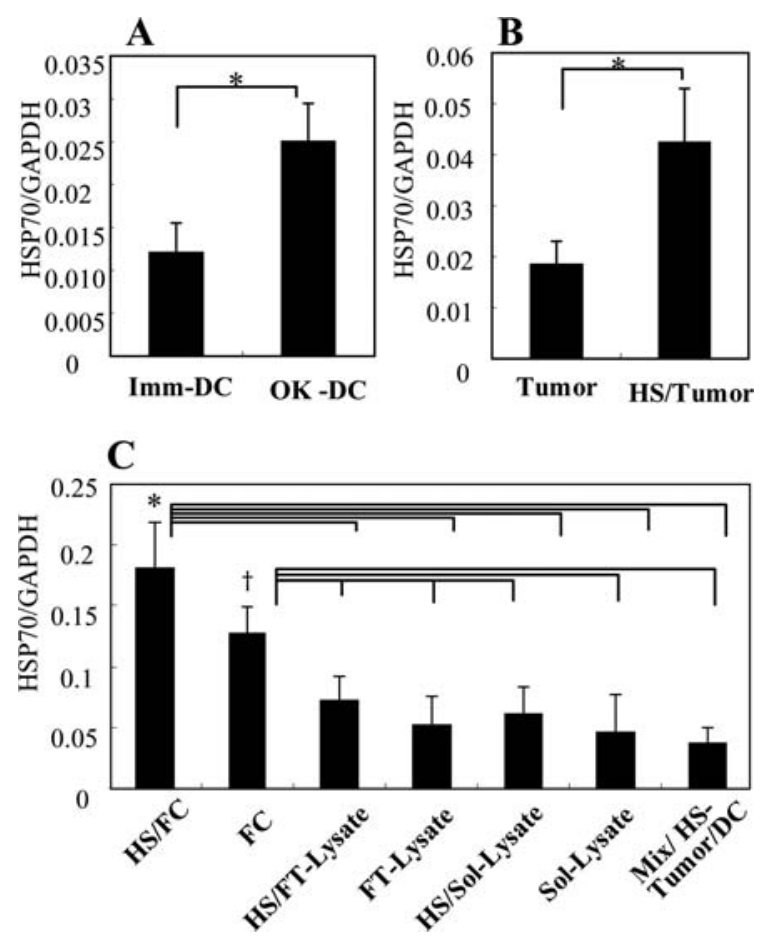

Figure 6. Comparison of HSP70 mRNA expression. (A) The ratios of copy numbers of HSP70 mRNA to GAPDH mRNA (HSP70/GAPDH) of OK432treated DC and immature DC were examined using real-time RT-PCR. ${ }^{*} \mathrm{p}<0.05$ (OK-DC vs. Imm-DC). (B) HSP70/GAPDH of HS/Tumor and Tumor was examined. ${ }^{*} \mathrm{p}<0.05$ (HS/Tumor vs. Tumor). (C) HSP70/GAPDH of HS/FC, FC, HS/FT-Lysate, FT-Lysate, HS/Sol-Lysate, Sol-Lysate and Mix/HS-Tumor/DC was examined. * $\mathrm{p}<0.05$ (HS/FC vs. all other groups except FC). ${ }^{\dagger} \mathrm{p}<0.05$ (FC vs. all other groups). Mean $\pm \mathrm{SD}(\mathrm{n}=10)$.

in all groups. $\mathrm{CD} 88^{+} \mathrm{T}$ cells stimulated with $\mathrm{FC}$ indicated significantly higher cytotoxicity than those of HS/FT-Lysate, FT-Lysate, HS/Sol-Lysate, Sol-Lysate, Mix/HS-Tumor/DC

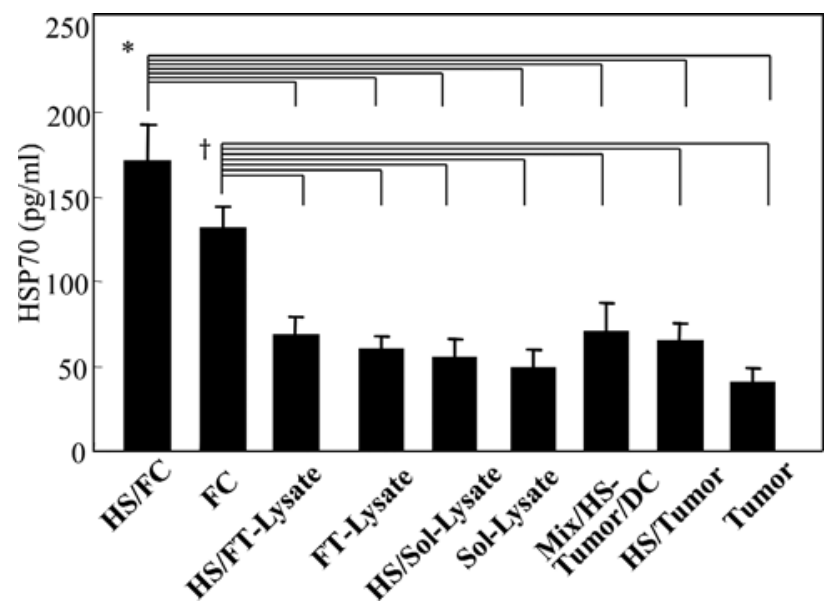

Figure 7. Production of HSP70 protein levels by ELISA. FCs (HS/FC, FC), tumor lysate-pulsed DCs (HS/FT-Lysate, FT-Lysate, HS/Sol-Lysate, SolLysate), Mix/HS-Tumor/DC, HS/Tumor and Tumor were cultured for 3 days and HSP70 protein levels of supernatants were examined by ELISA. ${ }^{*} \mathrm{p}<0.05$ (HS/FC vs. all groups except FC). ${ }^{\dagger} \mathrm{p}<0.05$ (FC vs. all groups except $\mathrm{HS} / \mathrm{FC})$. Mean $\pm \mathrm{SD}(\mathrm{n}=10)$.

(A) Wes tern blotting : HSP70
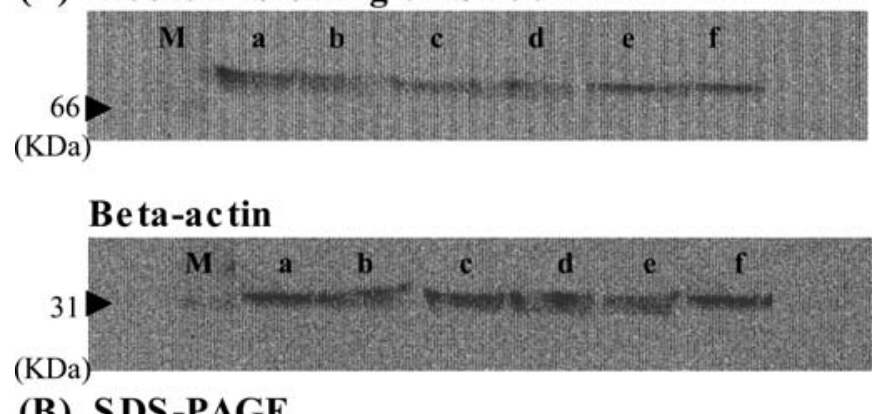

(B) SDS-PAGE

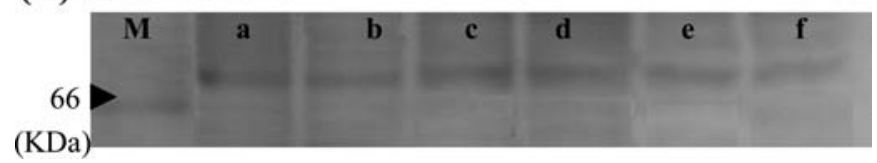

Figure 8. Detection of HSP70.PC by Western blotting and SDS-PAGE. The expressions of HSP70 with peptide complex (HSP70.PC) and ß-actin of immunoprecipitation samples in 8 groups were examined by Western blot analysis (A). Each lane show the molecule marker (M), HS/FC (a), FC (b), HS/Tumor (c), Tumor (d), HS/FT-Lysate (e), and HS/Sol-Lysate (f). After purification of immunoprecipitation samples by gel filtration chromatography, a portion was determined by SDS-PAGE (B). Each lane shows the molecule marker (M), HS/FC (a), FC (b), HS/Tumor (c), Tumor (d), HS/FTLysate (e), and HS/Sol-Lysate (f).

and medium. In contrast, all lysate groups (HS/FT-Lysate, FT-Lysate, HS/Sol-Lysate and Sol-Lysate groups) showed significant lower levels of CTL activities than Mix/HSTumor/DC and medium groups. Next, we examined MHC restriction of CTL induced by FCs and DCs pulsed with tumor lysates. Cytotoxicity against allogeneic MKN28 (HLAA*31012, HER-2 ${ }^{+}$) was not detected in any groups (Fig. 4B). Furthermore, CTL activities induced by HS/FC, FC, HS/FTLysate, FT-Lysate, HS/Sol-Lysate and Sol-Lysate groups were decreased by the addition of anti-MHC class I mAb but not anti-MHC class II mAb (Fig. 5). These results suggest that 

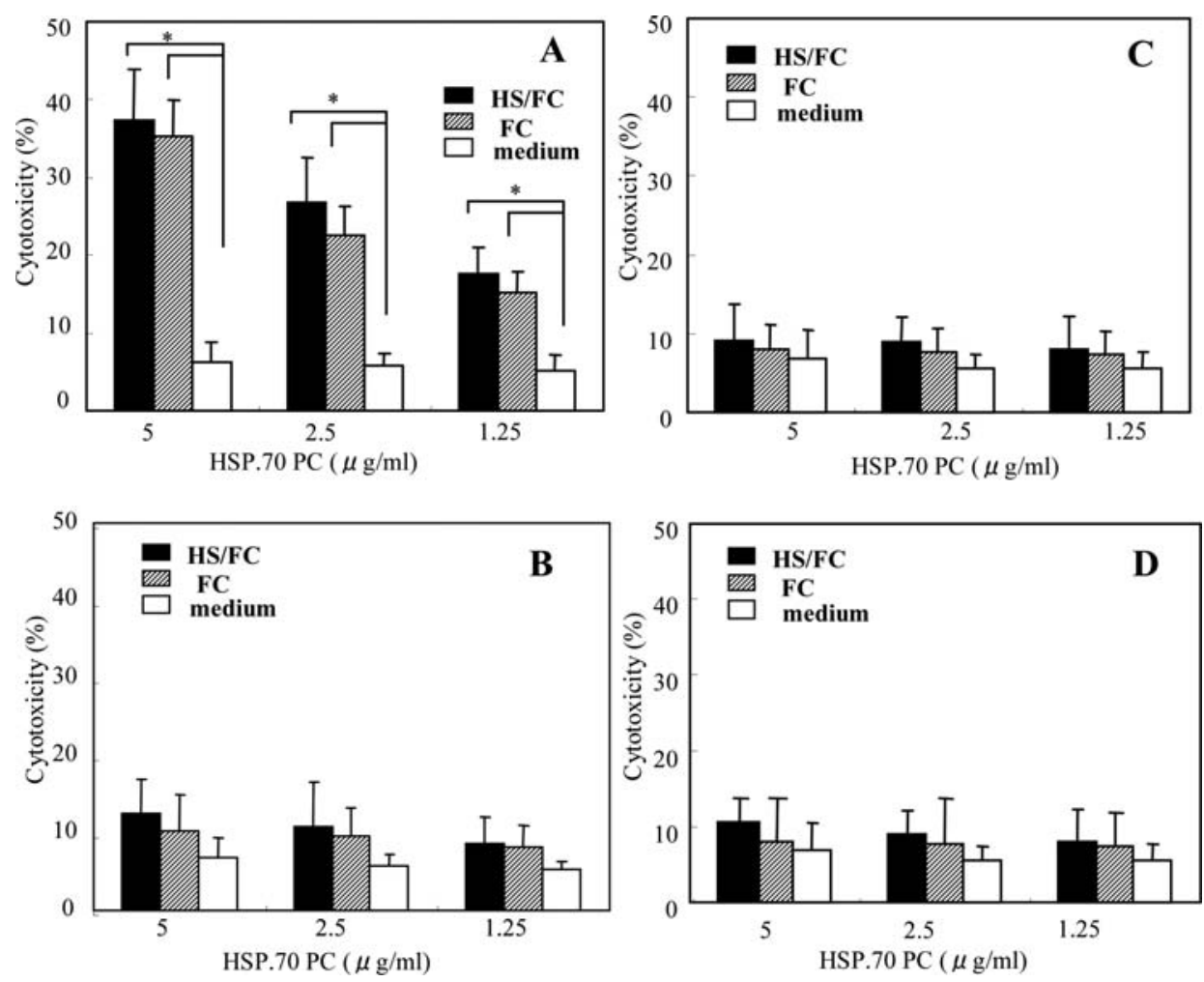

Figure 9. CTLs activity depend on the dose of HSP70.PC. CD8 ${ }^{+}$T cells from 5 patients were stimulated with autologous DCs pulsed with $5,2.5 \mathrm{or} 1.25 \mu \mathrm{g} / \mathrm{ml}$ of purified HSP70.PC extracted from HS/FC or FC. CTL activity against autologous tumor cells (A), allogeneic MKN28 tumor cells (B), autologous normal cells (C) and K562 cells (D) were examined by ${ }^{51} \mathrm{Cr}$ release assay. ${ }^{*} \mathrm{p}<0.05$ (HS/FC, FC vs. medium). Mean $\pm \mathrm{SD}(\mathrm{n}=5$ ).

FC possess stronger ability for MHC-restricted CTL production than DC loaded with soluble lysate or freeze-thawed tumor cell lysate. The modification of FC by heat stress could enhance the ability of CTL production.

Expression of HSP70 $m R N A$. The ratio of the HSP70 mRNA level to GAPDH level (HSP70/GAPDH) of OK-DC showed a significant increase compared to that of Imm-DC (Fig. 6A). HS-Tumor showed a significant increase of HSP70/GAPDH mRNA levels compared to the Tumor (Fig. 6B). Next, we examined the 8 groups of HSP70/GAPDH mRNA levels. HS-FC showed significantly the highest HSP70/GAPDH levels among the groups (Fig. 6C). HSP70/GAPDH levels of HS-FC and FC were significantly higher than those of HS/Sol-Lysate, Sol-Lysate, HS/FT-Lysate, FT-Lysate, DC/HS-Mixture and OK-DC. Our results suggest that FCs increase HSP70 mRNA levels as compared with DCs pulsed with tumor-soluble lysate and freeze-thawed lysate, and modification of FCs by heat stressing tumor cells increased the expression of HSP70 mRNA.

HSP70 protein levels by ELISA. HSP70 protein levels of $\mathrm{HS} / \mathrm{FC}$ and FC were significantly higher than those of HS/ FT-Lysate, FT-Lysate, HS/Sol-Lysate, Sol-Lysate, Mix/HSTumor/DC, HT/Tumor and Tumor (Fig. 7); HS/FC showed the highest protein levels in all groups. These results suggest that FCs increase HSP70 protein production more than DCs pulsed with tumor-soluble lysate and freeze-thawed lysate, and heat stressing tumor cells increased the production of HSP70 protein.
HSP70.PC levels by Western blotting. HSP70-peptide complex (HSP70-PC) separated by immunoprecipitation was purified by gel filtration chromatography, the expressions of HSP70.PC of HS-FC, FC, HS/Tumor, Tumor, HS/FT-Lysate and HS/SolLysate were examined by Western blot analysis (Fig. 8A). All samples showed a clear band of HSP70.PC and B-actin. Furthermore, immunoprecipitation samples were purified by gel filtration chromatography for CTL assay, and a portion of samples was examined for SDS-PAGE. In SDS-PAGE, clear band of HSP70.PC were detected in all samples.

Production of CTL activities by HSP70.PC-pulsed DCs. To examine the potential of HSP70.PC for CTL production, CD8 ${ }^{+}$ $\mathrm{T}$ cells from five patients with gastric cancer were stimulated with autologous DCs pulsed with three different doses of HSP70.PC $(5,2.5,1.25 \mu \mathrm{g} / \mathrm{ml})$ purified by gel filtration chromatography. HSP70.PC were prepared from autologous $\mathrm{HS} / \mathrm{FC}$ and FC, and CTL activities were examined against autologous tumor cells, allogeneic MKN28 tumor cells, autologous normal cell and K562 (Fig. 9). CTL activities against autologous tumor cells increased in a dose-dependent manner of HSP.70 PC (Fig. 9A). In contrast, allogeneic MKN28 and K562 cells did not show any CTL activities (Fig. 9B and C), and autologous normal cells also did not show cytotoxic activities (Fig. 9D).

Next, using the same volume of HSP70.PCs $(5 \mu \mathrm{g} / \mathrm{ml})$, CTL productivity of DCs pulsed with HSP70.PC was compared. HSP70.PC were prepared from 7 groups (Fig. 10). CTL activities against autologous tumor cells induced by DCs pulsed with HSP70.PC of HS/FC were significantly higher 

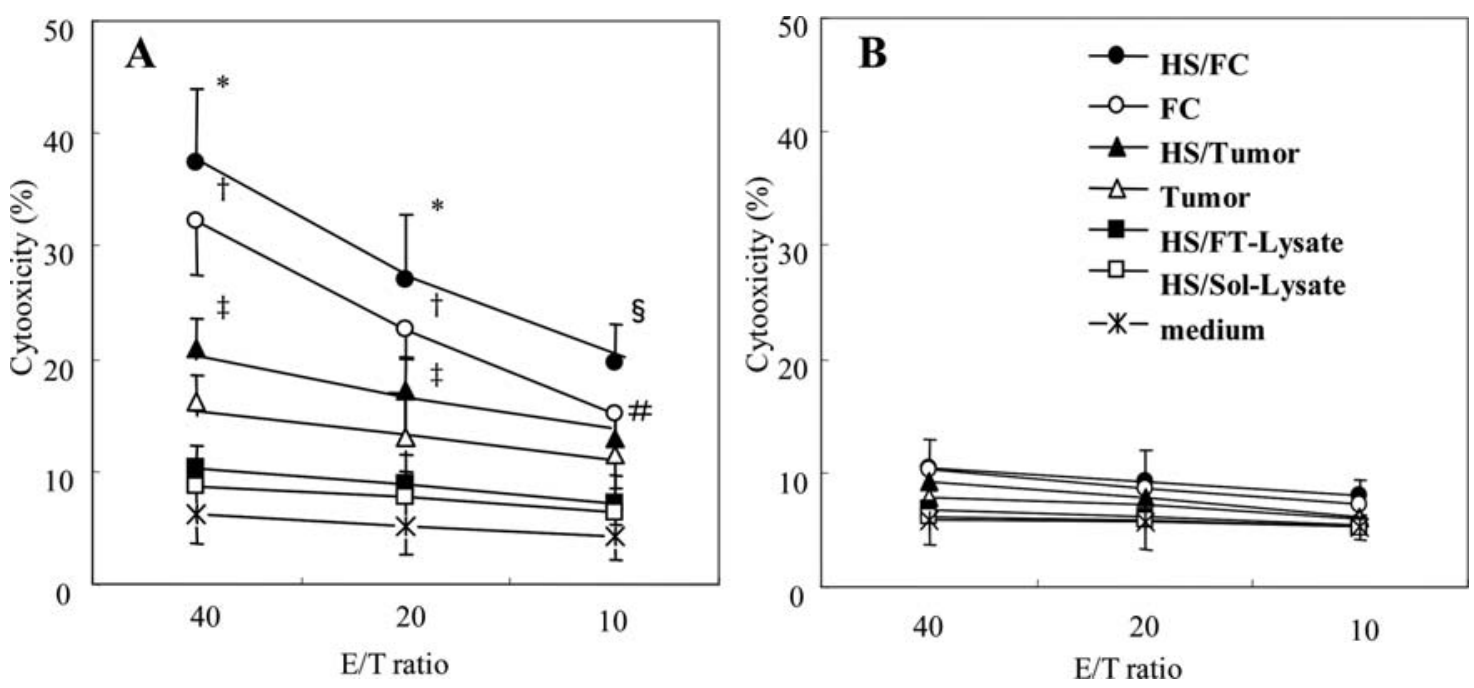

Figure 10. CTL activity by HSP70.PC-pulsed DC. To evaluate the potential of HSP70.PC for CTL production, CD8 ${ }^{+} \mathrm{T}$ cells from 5 patients were co-cultured with DC pulsed with $5 \mu \mathrm{g} / \mathrm{ml}$ of HSP70PC, and cytotoxicities against autologous tumor cells (A) and allogeneic MKN28 cells (B) were examined by ${ }^{51} \mathrm{Cr}$ release assay. HSP70.PC were extracted from HS/FC, FC, HS/Tumor, Tumor, HS/FT-Lysate, HS/Sol-Lysate. " $p<0.05$ (HS/FC vs. HS/Tumor, Tumor, HS/FTLysate, HS/Sol-Lysate and medium), ${ }^{\dagger} \mathrm{p}<0.05$ (FC vs. Tumor, HS/FT-Lysate, HS/Sol-Lysate and medium), ${ }^{\star} \mathrm{p}<0.05$ (HS/Tumor vs. HS/FT-Lysate, HS/SolLysate and medium), ${ }^{8} \mathrm{p}<0.05$ (HS/FC vs. Tumor, HS/FT-Lysate, HS/Sol-Lysate and medium) ${ }^{*} \mathrm{p}<0.05$ (FC vs HS/FT-Lysate, HS/Sol-Lysate and medium). Mean $\pm \operatorname{SD}(n=5)$.

than those of HS/Tumor, Tumor, HS/FT-Lysate, HS/SolLysate and medium. DC pulsed with HSP70.PC of FC also showed significant higher CTL productivities than those of Tumor, HS/FT-Lysate, HS/Sol-Lysate and medium. HS/FC showed the highest cytotoxicity in all groups. HS/Tumor group induced significant CTL activities; however, these CTL levels were lower than those of FC groups. In contrast, DCs pulsed with HSP70.PC of HS/FT-Lysate or HS/Sol-Lysate did not induce CTL activity. Next, CTL activity against allogeneic MKN28 cells was examined, and it was clarified that $\mathrm{CD}^{+} \mathrm{T}$ cells stimulated with DCs pulsed with HSP70. PC in all 7 groups did not show distinct CTL activity (Fig. 10B). The present results suggest that DCs pulsed with HSP70.PC extracted from HS/FC and FC possess strong ability for CTL production but not NK and autoimmune responses; however, DCs pulsed with HSP70.PC of tumor lysate-loaded DCs did not induce CTL activity.

\section{Discussion}

The present studies demonstrated that CTL productivity of FCs is superior to that of DCs pulsed with soluble and freezethawed tumor lysates, and it is suggested that up-regulation of HSP70.PC was related to the excellent CTL productivity of FCs.

Abundant experimental evidence indicates that DCs loaded with tumor-associated antigens stimulate anti-tumor immune responses; however, a significant percentage of patients with advanced or metastatic tumors respond poorly to DC-based immunotherapy, as well as adjuvant therapy such as chemotherapy. To increase the efficacy of DC-based immunotherapy, improvement and optimization of vaccine, including the antigen-loading method, are desired. Using the mouse model, a few studies compared the immunotherapeutic reactivity of DCs loaded with a variety of antigen preparation, and demonstrated that the FCs of DCs with tumor cells generated a superior anti-tumor effect to other antigen loading methods $(4,5)$. However, in human model, there is considerable controversy as to which antigen-loading methods represent the most immunogenic forms of vaccines that are capable of eliciting clinically relevant therapeutic immunity. In our study using autologous DCs and tumor cells from patients with gastric cancer, we compared FCs and DCs pulsed with two types of tumor lysate, because these methods are one of the most popular methods and is used in clinical trials. Our present results demonstrated that FCs possess significantly higher ability for MHC class I restricted CTL production than those of DCs loaded with soluble or freeze-thawed lysate and modification of FCs by heat stress enhanced the ability of CTL production. To the best of our knowledge, the present study is the first to compare the modified cell fusion and tumor lysate using autologous DCs and tumor cells in patients with gastric cancer.

FCs from tumor cells and DCs possess both the cell components of tumor antigens and the accessory molecules necessary for antigen processing and presentation. Previous studies have demonstrated that FCs of tumor cells and DCs create an immunogenic cell by cytoplasm integration between the respective cells. Such integration makes it possible to exchange cytoplasmic contents and share cellular compartments. Our own and other studies have shown the coexpression of tumor-associated antigens and cell-surface molecules of DCs on FCs (4-7). In the present study, we modified FCs by heat stress of tumor cells and maturation of DC by OK-432. The streptococcal preparation OK432, which is a TLR4 agonist, is a commercially available good manufacturing practice grade immunomodulatory agent which has been widely used for cancer patients. We have reported that OK432 possesses strong ability for DC maturation (24). It is known that heat treatment of tumor cells could increase the expression of tumor antigens such as MUC1 (23). Our present data demonstrated that FCs of heat-stressed tumor cells and 
OK-stimulated DC significantly increased CEA and HER-2 tumor antigens and co-stimulated molecules such as CD80, CD83 and CD88, suggesting the enhanced immunogenicity of these modified FCs.

Next, we examined in more detail the superior CTL production of FCs. It was thought that there are several potential mechanisms for FCs to induce an effective CTL response to tumor antigens. Direct antigen presentation is consistent with DC characteristics; however, other mechanisms, such as crosspresentation, adjuvant activity and bystander effects, may also have important roles. It is known that HSPs can induce the cross-presentation of immunocomplex of tumor antigens. The promising potential of a molecular-chaperone-based tumor vaccine using the immunocomplex combined with HSP has been the focus of much attention; however, research into the mechanisms underlying the effect of HSP on immune cells is only at an early stage. By differences in molecule weight, HSPs are classified into families, such as HSP40, 60, 70, 90 and 110. In these HSP families, HSP70 is one of the important molecules because of its strong binding of the tumor antigen peptide, efficient uptake of HSP70 through the receptors, and activation of an immune response (18-22). HSP70 binds effectively to LOX-1, a member of C-type lectin receptors which is found on the DC cell surface, and signaling through LOX-1, including the activation of TLR-2 and innate immunity (25-27). It is known that at least 12 HSP70 genes are contained within human cells, and three (HSP-70-1a, HSP70-1b, HSP70-HOM) of these genes are encoded by genes within the MHC locus, suggesting a connection to immune function (28-32). Our results showed a significant upregulation of HSP.70 mRNA and protein levels in FCs as compared with those of DCs pulsed with soluble tumor cell lysates or freeze-thawed tumor cell lysates. Furthermore, mRNA and the protein level of HSP70 of FCs increased significantly by heat stressing tumor cells. A separate study in colorectal cancer, has also demonstrated an increase of HSP70 protein levels in HS/FC, thereby supporting our results (23).

To identify the potential of HSP70.PC for CTL production, we isolated HSP70.PC from FCs by immunoprecipitation and purified them with fluid chromatography. Our present results demonstrate that DCs pulsed with HSP70.PC of FCs induced significantly higher levels of CTL activities than DCs pulsed with HSP70.PC or autologous tumor cell lysates. Using the mouse model, it has been reported that HSP70.PC of FCs stimulated $\mathrm{T}$ cell proliferation in vitro (18). However, to our knowledge, the present study is the first to demonstrate the superior CTL productivity of HSP70.PC prepared from heatstressed FCs of gastric cancer patients.

In conclusion, we demonstrated the strong potential of an FC vaccine for CTL production, and this process may be related to the up-regulation of HSP70. The molecular mechanism of FCs that promotes the formation of immunogenic complexes of HSP with tumor antigenic peptides is complex, and we need further study to clarify the process.

\section{Acknowledgements}

We are grateful to Dr K. Okinaga and Dr. J. Gong for their comments. We thank Ms. J. Tamura for her technical support.
This study was supported by grants from the Ministry of Education, Science and Culture (Grant-in-Aid C 19591494).

\section{References}

1. Inaba K, Metlay JP, Crowley MT, Witmer-Pack M and Steinman RM: Dendritic cells as antigen presenting cells in vivo. Int Rev Immunol 6: 197-206, 1990.

2. Apostolopolus V, Weiner DB and Gong J: Cancer vaccines: methods for inducing immunity. Expert Rev Vaccines 8: 861-862, 2008.

3. Tacken PJ, De Vries IJ, Torensma R and Figdor CG: Dectritic immunotherapy: from ex vivo loading to in vivo targeting. Nat Rev Immunol 7: 790-802, 2007

4. Yasuda T, Kamigaki T, Nakamura T, et al: Dendritic cell-tumor cell hybrids enhance the induction of cytotoxic T lymphocytes against murine colon cancer: A comparative analysis of antigen loading methods for the vaccination of immunotherapeutic dendritic cells. Oncol Rep 16: 1317-1324, 2006.

5. Shimizu K, Kuriyama H, Kjaergaard J, Lee W, Tanaka H and Shu S: Comparative analysis of antigen loading strategies of dendritic cells for tumor immunotherapy. J Immunother 27: 265-272, 2004.

6. Ogawa F, Iinuma $\mathrm{H}$ and Okinaga $\mathrm{K}$ : Dendritic cell vaccine therapy by immunization with fusion cells of interleukin-2 gene-transduced, spleen-derived dendritic cells and tumour cells. Scand J Immunol 59: 432-439, 2004

7. Iinuma H, Okinaga K, Fukushima R, et al: Superior protective and therapeutic effects of IL-12 and IL-18 gene-transduced dendritic neuroblastoma fusion cells on liver metastasis of murine neuroblastoma. J Immunol 176: 3461-3469, 2006.

8. Takeda A, Homma S, Okamoto T, Kufe D and Ohno T: Immature dendritic cell/tumor cell fusions induce potent antitumour immunity. Eur J Clin Invest 33: 897-904, 2003.

9. Tanaka Y, Koido S, Ohana M, Liu C and Gong J: Induction of impaired antitumor immunity by fusion of MHC class IIdeficient dendritic cells with tumor cells. J Immunol 174: 1274-1280, 2005

10. Iinuma $\mathrm{H}$ and Okinaga $\mathrm{K}$ : Cancer immunotherapy based on DCtumor fusion vaccine. Biotherapy 21: 48-55, 2007.

11. Krause SW, Neumann C, Soruri A, et al: The treatment of patients with disseminated malignnant melanoma by vaccination with autologous cell hybrids of tumor cells and dendritic cells. J Immunother 25: 421-428, 2002.

12. Marten A, Renoth S, Heinicke T, et al: Allogeneic dendritic cells fused with tumor cells:preclinical results and outcome of a clinical phase I/II trial in patients with metastatic renal cell carcinoma. Hum Gene Ther 14: 483-494, 2003.

13. Trefzer U, Herberth G, Wohlan K, et al: Vaccination with hybrids of tumor and dendritic cells induces tumor-specific T-cell and clinical responses in melanoma stage III and IV patients. Int J Cancer 110: 730-740, 2004.

14. Kikuchi T, Akasaki Y, Abe T, et al: Vaccination of glioma patients with fusions of dendritic and glioma cells and recombinant human interleukin 12. J Immunother 27: 452-459, 2004.

15. Haenssle HA, Krause SW, Emmer S, et al: Hybrid cell vaccination in metstatic melanoma: clinical and immunologic results of a phase I/II study. J Immunother 27: 147-155, 2004.

16. Homma S, Kikuchi T, Ishiji N, et al: Cancer immunotherapy by fusions of dendritic and tumor cells and rh-IL-12. Eur J Clin Invest 35: 279-286, 2005.

17. Avigan DE, Vasir B, George DJ, et al: Phase I/II study of vaccination with electrofused allogeneic dendritic cells/ autologous tumor-derived cells in patients with stage IV renal cell carcinoma. J Immunother 30: 749-761, 2007.

18. Enomoto Y, Bharti A, Khaleque AA, et al: Enhanced immunogenicity of heat shock protein 70 peptide complexes from dendritic cell-tumor fusion cells. J Immunol 177: 5946-5955, 2006.

19. Kurotaki T, Tamura Y, Ueda G, et al: Efficient cross-presentation by heat shock protein 90 -peptide complex-loaded dendritic cells via an endosomal pathway. J Immunol 179: 1803-1813, 2007.

20. Udono H and Srivastava PK: Heat shock protein 70-associated peptides elicit specific cancer immunity. J Exp Med 178: 1391-1396, 1993.

21. Castelli C, Ciupitu AM, Rini F, et al: Human heat shock protein 70 peptide complexes specifically activate antimelanoma $\mathrm{T}$ cells. Cancer Res 61: 222-227, 2001. 
22. Bendz H, Ruhland SC, Pandya MJ, et al: Human heat shock protein 70 enhances tumor antigen presentation through complex formation and intracellular antigen delivery without innate immune signaling. J Biol Chem 282: 31688-31702, 2007.

23. Koido S, Hara E, Homma S, et al: Synergistic induction of antigen-specific CTL by fusions of TLR-stimulated dendritic cells and heat-stressed tumor cells. J Immunol 179: 4874-4883, 2007.

24. Ogihara T, Iinuma $\mathrm{H}$ and Okinaga $\mathrm{K}$ : Usefulness of immunomodulators for maturation of dendritic cells. Int J Oncol 25 : 453-459, 2004.

25. Jin G, Kubo H, Kashiba M, et al: Saposin B is a Human coenzyme Q10-binding/transfer protein. J Clin Biochem Nutr 42: 167-174, 2008

26. Vabulas RM, Ahmad-Nejad P, Ghose S, Kirschning CJ, Issels RD and Wagner H: HSP70 as endogenous stimulus of the toll/ interleukin-1 receptor signal pathway. J Biol Chem 277: 15107-15112, 2002

27. Delneste Y, Magistrelli G, Gauchat J, et al: Involvement of LOX-1 in dendritic cell-mediated antigen cross-presentation. Immunity 17: 353-362, 2002.
28. Binder RJ, Vatner R and Srivastava P: The heat-shock protein receptors: some answers and more questions. Tissue Antigens 64: 442-451, 2004

29. Thériault JR, Mambula SS, Sawamura T, Stevenson MA and Calderwood SK: Extracellular HSP70 binding to surface receptors present on antigen presenting cells and endothelial/ epithelial cells. FEBS Lett 579: 1951-1960, 2005.

30. Bukau B and Horwich AL: The Hsp70 and Hsp60 chaperone machines. Cell 92: 351-366, 1998

31. Tang D, Khaleque MA, Jones EL, et al: Expression of heat shock proteins and heat shock protein messenger ribonucleic acid in human prostate carcinoma in vitro and in tumors in vivo. Cell Stress Chaperones 10: 46-58, 2005.

32. Fourie AM, Peterson PA and Yang Y: Characterization and regulation of the major histocompatibility complex-encoded proteins Hsp70-Hom and Hsp70-1/2. Cell Stress Chaperones 6: 282-295, 2001. 